\title{
Cardiovascular conditions and COVID-19
}

\section{Atifur Rahman}

ALTHOUGH Covid-19 is caused by the severe acute respiratory syndrome coronavirus 2 (SARS-CoV-2) and primarily affects the respiratory system, cardiovascular involvement is common, especially in individuals who are severely ill.

The cardiovascular manifestations reported to be directly related to COVID-19 include myocardial injury, severe myocarditis/myopericarditis, pericardial effusion, acute coronary syndrome, cardiomyopathy, heart failure, cardiogenic shock, supraventricular and ventricular arrhythmias and thromboembolic disease including deep vein thrombosis and pulmonary embolism.

\section{Elevated biomarkers: Troponin and natriuretic peptides}

For patients who are hospitalised with COVID-19, mild elevation of troponin is common (19.7\%) and frequently correlates with disease severity, acting as a marker for cardiac injury. ${ }^{1}$

The cause of troponin elevation in serious infection is multifactorial. It has been suggested that inflammatory cytokines, particularly tumour necrosis factor alpha (TNF- $\alpha$ ) and interleukin 6 (IL-6), are responsible for direct myocardial depression and increased cell membrane permeability to troponin molecules. Decreased myocardial perfusion due to hypotension, increased oxygen consumption due to tachycardia, release of catecholamine with subsequent vasoconstriction and increased coagulation of the capillary bed have all been proposed to play a part in myocyte damage and troponin release. ${ }^{2}$

A recent statement by the American College of Cardiology suggests that a rise and/or fall of high-sensitivity troponin is not sufficient to secure the diagnosis of acute myocardial infarction, which should be based on clinical judgment, symptoms/ signs and electrocardiographic changes. Investigations including echocardiography or coronary angiography for patients with COVID-19 who have elevated biomarkers should be restricted to patients in which such procedures are expected to influence clinical decision making. ${ }^{3}$

Natriuretic peptides (B-type natriuretic peptide [BNP] or N-terminal pro BNP [NT-proBNP]) are biomarkers of myocardial stress. They are frequently elevated among patients infected with SARS-CoV-2 with severe respiratory illnesses, typically in the absence of elevated filling pressures or clinical heart failure. ${ }^{3}$ Similar to troponin, elevation of BNP or NT-proBNP is associated with unfavourable outcomes for patients with acute respiratory distress syndrome (ARDS).

\section{Acute coronary syndrome}

It is possible for both type 1 myocardial infarction (due to a plaque rupture) and type 2 myocardial infarction (due to myocardial supply/demand mismatch because of tachycardia, hypoxia from ARDS and potential microthrombi) to be triggered by COVID-19. ${ }^{3}$

Case reports describing patients with COVID-19 presenting with electrocardiogram findings suggesting an ST-elevation myocardial infarction or non-ST elevation myocardial infarction without angiographic evidence of obstructive coronary artery disease (CAD) have been published. ${ }^{4}$

According to recently released consensus guidelines from the Cardiac Society of Australia and New Zealand, thrombolytic therapy may be considered (preferred) for patients with suspected
COVID-19 and an ST-elevation myocardial infarction. ${ }^{5}$

There is no evidence to support the use of early invasive coronary angiography among patients with type 2 myocardial infarction. During the pandemic, strict infection control measures to limit infection spread within the hospital and to healthcare workers should be an important consideration.

No data exist to suggest benefit from antiplatelet or anticoagulant therapy for patients with acute myocardial injury due to type 2 myocardial infarction. ${ }^{3}$

\section{Cardiomyopathy and heart failure}

Among patients who are hospitalised and critically ill with COVID-19, cardiomyopathy and associated heart failure and cardiogenic shock have been reported. ${ }^{4}$ These patients have an extremely high mortality rate and usually require cardiopulmonary support.

A potential mechanism of cardiac involvement in COVID-19 is direct myocardial involvement mediated via angiotensin-converting enzyme 2 (ACE2). Given increased interleukin levels are frequently observed in such patients, it is also possible that the mechanisms of cardiac involvement include a cytokine storm due to hyperinflammation. ${ }^{6}$

\section{Treatment of hypertension and coronary artery disease with angiotensin-converting enzyme inhibitors or angiotensin receptor blockers}

Hypertension and CAD are common among patients with COVID-19 and are associated with a poorer clinical prognosis. Both hypertension and CAD are often treated with angiotensin-converting enzyme (ACE) inhibitors or angiotensin 
receptor blockers (ARBs). Infection with SARS-CoV-2 is caused by the binding of a spike protein on a viral surface to the ACE2 receptor. There is concern that treatment with an ACE inhibitor or ARB could increase a patient's susceptibility to the virus by upregulating ACE2.

The European Society of Cardiology council released a statement on hypertension, ACE inhibitors and ARBs, stating they 'strongly recommend that physicians and patients should continue treatment with their usual anti-hypertensive therapy because there is no clinical or scientific evidence to suggest that treatment with ACE inhibitors or ARBs should be discontinued because of the COVID-19 infection'. The American College of Cardiology and American Heart Association have also advocated this.

\section{Conclusion}

Common cardiac manifestations of SARS-CoV-2 infection include myocardial injury, myocarditis, heart failure, arrhythmias and myocardial infarction. Pre-existing cardiovascular disease is also associated with an increased risk of mortality among patients hospitalised with COVID-19. Data on the interaction between the cardiovascular system and SARS-CoV-2 are still being explored. Ongoing studies currently in progress will provide a greater understanding in the future.

\section{Key points}

- Hypertension and underlying CAD are common among patients with COVID-19. There is no evidence to suggest that treatment with ACE inhibitors or ARBs should be discontinued because of SARS-CoV-2 infection.

- Elevation biomarkers such as troponin and natriuretic peptides are common in patients hospitalised with COVID-19 and frequently correlate with disease severity.

- Myocardial infarctions due to a plaque rupture (type 1 myocardial infarction) or myocardial supply/demand mismatch (type 2 myocardial infarction) triggered by COVID-19 infection have been reported. Investigations in such patients should be restricted to situations when procedures are expected to influence the clinical decision making. Strict infection control measures to limit infection spread should be an important consideration.

- Data do not support the routine use of antiplatelet or anticoagulant therapy for patients with acute myocardial injury due to supply/demand mismatch.

- For patients who are critically ill and hospitalised with COVID-19, cardiomyopathy and associated heart failure and cardiogenic shock have been reported. These patients have an extremely high mortality rate.

First published online 20 May 2020.

\section{Author}

Atifur Rahman MBBS, FRACP, FCSANZ, Interventional Cardiologist, Gold Coast University Hospital, Qld; Associate Professor, School of Medicine, Griffith University, Qld. atifur@hotmail.com Competing interests: None.

Provenance and peer review: Not commissioned, peer reviewed.

Citation: Rahman A. Cardiovascular conditions and COVID-19. Aust J Gen Pract 2020;49 Suppl 22. doi: 10.31128/AJGP-COVID-22.

\section{References}

1. Shi S, Qin M, Shen B, et al. Association of cardiac injury with mortality in hospitalized patients with COVID-19 in Wuhan, China. JAMA Cardiol 2020;e200950. doi: 10.1001/ jamacardio.2020.0950

2. Kalla C, Raveh D, Algur N, Rudensky B, Yinnon AM, Balkin J. Incidence and significance of a positive troponin test in bacteremic patients without acute coronary syndrome. Am J Med 2008;121(1):909-15. doi: 10.1016/j.amjmed.2008.05.037.

3. Januzzi JL Jr. Troponin and BNP use in COVID-19. American College of Cardiology. 18 March 2020. Available at www.acc.org/latest-in-cardiology/ articles/2020/03/18/15/25/troponin-and-bnpuse-in-covid19 [Accessed 13 May 2020].

4. Baliga RR. Coronavirus fulminant myocarditis case review. American College of Cardiology. 18 March 2020. Available at www.acc.org/latest-in-cardiology/tenpoints-to-remember/2020/03/18/12/00/ coronavirus-fulminant-myocarditis-saved [Accessed 13 May 2020].

5. Lo STH, Yong AS, Sinhal A. Consensus guidelines for interventional cardiology services delivery during COVID-19 pandemic in Australia and New Zealand. Heart Lung Circ 2020. doi: 10.1016/j. hlc.2020.04.002.

6. Puja M, McAuley DK, Brown M, et al COVID-19: Consider cytokine storm syndromes and immunosuppression. Lancet 2020;395(10229):1033-34. doi: 10.1016/S0140 6736(20)30628-0. 